\title{
Reduced damage threshold for tungsten using combined steady state and transient sources
}

\author{
T.W. Morgan ${ }^{\mathrm{a}^{*}}$, J.J. Zielinski ${ }^{\mathrm{a}}$, B.J. Hensen ${ }^{\mathrm{a}}$, H.Y. Xu ${ }^{\mathrm{b}}$, L. Marot ${ }^{\mathrm{c}}$, and G. De Temmerman ${ }^{\mathrm{a}}$ \\ ${ }^{a}$ FOM Institute for Plasma Physics, Rijnhuizen, Edisonbaan 14, 3439 MN Nieuwegein, The Netherlands \\ ${ }^{b}$ Department of materials science and engineering, Tsinghua University, Beijing 100084, China \\ ${ }^{c}$ Department of Physics, University of Basel, Klingelberstrasse 82, CH-4056 Basel, Switzerland
}

\begin{abstract}
Divertor wall materials in future fusion devices will be subject to both high flux steady state plasma and transient ELM striking which could limit the lifetime of these plasma facing materials. A pulsed plasma source was therefore developed to reproduce these conditions. Laser irradiation of similar pulse length has been used to disambiguate between the effects of particle and heat loads compared with transient heating alone. A lowered threshold for damage of tungsten was observed in the case of simultaneous transient and steady state loads compared to transients alone, while surface damage was also enhanced with repeated laser irradiation following steady plasma exposure, compared to the two processes individually, indicating a synergistic enhancement of surface modification due to plasma exposure.
\end{abstract}

PACS: 52.40.Hf, 52.38.Mf, 52.50.Dg

PSI-20 keywords: Bubbles and blisters, ELM, Laser, Melting, Tungsten

* Corresponding/presenting author postal address: Dutch Institute for Fundamental Energy Research (DIFFER), PO Box 1207, 3430 BE Nieuwegein, The Netherlands

Corresponding/presenting author email address: t.w.morgan@differ.nl 


\section{Introduction}

Erosion, cracking and melting of plasma facing materials (PFMs) by Edge Localised Modes (ELMs) provide a significant threat to their expected lifetime as well as concerns for plasma performance from the introduction of impurities to the core plasma. Energy densities of unmitigated type-I ELMs are predicted to be up to $10 \mathrm{MJ} \mathrm{m}^{-2}$ [1] while a steady state heat flux of around $10 \mathrm{MW} \mathrm{m}^{-2}$ is also expected to strike the divertor targets [2] [2]. The steady state flux can produce deformations of the material surface, such as blistering [3] and heliuminduced nano-structures [4] [5], and these changes in morphology could affect the material response during an ELM. The synergistic effect of repeated ELM striking in conjunction with this steady state flux is therefore a topic of considerable interest. However, there are few experiments [6] [7] which replicate the combination of steady state heat loads and both transient particle and heat loads expected in ITER. A device capable of delivering this combined load has therefore been developed while a millisecond pulse laser has also been used to assist in disambiguating the effect of heat loading alone from combined heat and particle loading. This paper presents a summary of the ongoing efforts utilising these two techniques.

\section{Development of a pulsed plasma source}

Cascaded arc discharge sources are an efficient source of dense plasma of moderate temperature, based upon electrical discharge through an injected gas confined to a channel bound by individually insulated conducting plates [8]. The source utilised here is designed for the investigation of fusion-relevant plasma-surface interactions [9] and was installed at the Pilot-PSI linear plasma device. In combination with the steady state current supply to the 
source, a capacitor bank ( $8400 \mu \mathrm{F}$, with up to $4.2 \mathrm{~kJ}$ stored energy) was connected which could be discharged through the source simultaneously with the DC supply [10] [11]. This combination of the steady plasma flux with a transient increase in output permitted an approximation of the simultaneous effect of steady state divertor plasma and ELM striking on tungsten within a single plasma source.

The capacitor discharge induces an increase of both voltage and current in the source of up to $14 \mathrm{kA}$ and $450 \mathrm{~V}$, compared with typical values of $0.2 \mathrm{kA}$ and $90 \mathrm{~V}$ in DC operation, with a typical pulse duration of $1.3 \mathrm{~ms}$. This results in a rise time of typically 0.6 $\mathrm{ms}$ for the pulse, which is similar to that expected for type I ELMs in ITER (0.1 to $1 \mathrm{~ms}$ [12]). In both steady and transient cases the beam density and temperature peak at the central axis and can be described with a Gaussian radial profile of $\sim 10 \mathrm{~mm}$ FWHM. Typical performance in DC operation in a $1.6 \mathrm{~T}$ field provides a peak temperature and density of $\sim 2.6 \mathrm{eV}$ and $\sim 7 \times 10^{20} \mathrm{~m}^{-3}$, respectively, measured $4 \mathrm{~cm}$ downstream from the source. By comparison during the pulse the peak electron temperature and density increase with input power up to $15 \mathrm{eV}$ and $8 \times 10^{21} \mathrm{~m}^{-3}$ at $2 \mathrm{MW}$ peak input power, an increase by a factor of $\sim 6$ and $\sim 11$ respectively (figure 1).

\section{Synergistic effects of combined transient and steady state plasma exposure}

Initial experiments combining pulsed and steady state plasmas as described in section 2 have been reported in detail in [13]. For the purpose of comparison with section 4 a summary of the relevant results is presented here.

$30 \mathrm{~mm}$ diameter, $1 \mathrm{~mm}$ thick, polycrystalline tungsten targets $(99.97 \%$ pure rolled tungsten manufactured by Plansee SE, Austria) were polished and exposed to steady state 
plasmas of hydrogen. The targets were kept at floating potential while being actively water cooled to maintain a steady surface temperature of $400^{\circ} \mathrm{C}$ during DC operation.

Samples were exposed to repeated combined pulsing (10 or 17 pulses) at different energy densities $\left(0.07,0.15\right.$ or $\left.0.50 \mathrm{MJ} \mathrm{m}^{-2}\right)$ with a steady state exposure time of $4 \mathrm{~s}$ per pulse. Reference samples were exposed to the same steady state plasma fluence for comparison (exposure time $40 \mathrm{~s}$ or $68 \mathrm{~s}$ for 10 or 17 pulses respectively, peak $n_{e} \sim 5 \times 10^{20} \mathrm{~m}^{-3}$ and $T_{e} \sim 1$ eV). SEM analysis of the target centres (those areas exposed to peak $n_{e}$ and $T_{e}$ ) shows that no morphology changes from an unexposed target were observed for the reference samples, but that roughening was observed after only 10 pulses at $0.07 \mathrm{MJ} \mathrm{m}^{-2}$ (equivalent to $\sim 2 \mathrm{MW} \mathrm{\textrm {m } ^ { - 2 }}$ $s^{1 / 2}$ per pulse). The amount of roughening increased both with energy density (figure 2) and pulse number. Burst blister caps were observed for the highest energy densities (figure 2d). Furthermore cracking is observed for all samples subjected to combined pulsing.

\section{Laser induced transient heat loads on pre-exposed targets}

In order to differentiate between the effects of transient heating and that of combined particle and heat fluxes, pulsed laser irradiation of tungsten targets simultaneously with exposure to steady state plasma is being developed at Magnum-PSI [14]. To benchmark the effects of these two elements separately tungsten targets (similarly prepared to those in section 2) pre-exposed to deuterium plasma in Pilot-PSI were irradiated by multiple laser pulses. The steady state exposures were made with similar conditions as above (peak $n_{e} \sim(1.6-$ 3.2) $\times 10^{20} \mathrm{~m}^{-3}$ and $\left.T_{e} \sim 0.8-1.4 \mathrm{eV}\right)$. Bias of $-38 \mathrm{~V}$ was applied to the surface to induce surface deformations such as blistering, unlike in section 3. The surface temperature during steady state exposure was measured by an IR camera to be $\sim 200{ }^{\circ} \mathrm{C}$. 
The laser pulse spot FWHM was $2.1 \pm 0.2 \mathrm{~mm}$ diameter with a duration of $1.2 \pm 0.2$ $\mathrm{ms}$ at $1064 \mathrm{~nm}$ from an Nd:YAG laser, with a repetition rate of $10 \mathrm{~Hz}$. This approximates more closely to the duration and periodicity of type I ELMs, compared to previous experiments investigating ELM-like heat-loads with nanosecond laser pulses [15] [16] [17]. A consequence of these longer exposures is that the characteristic heat deposition depth is increased from $\sim 1 \mu \mathrm{m}$ to $\sim 500 \mu \mathrm{m}$ lengths for these ELM-length pulses.

After plasma exposure the samples were laser irradiated in a $\sim 3 \mathrm{~m}^{3}$ vacuum vessel, while the target surface was observed by fast IR imaging for surface temperature. Molecular deuterium desorbed due to the laser pulses was monitored by a QMS-analyser. Samples were uncooled and initially at $22{ }^{\circ} \mathrm{C}$.

Samples were exposed to different fluences between $\Phi=0.6 \times 10^{25}$ and $11.2 \times 10^{25} \mathrm{D}^{+} \mathrm{m}^{-}$ ${ }^{2}$ (see figure 4) Pre-exposed samples displayed clear blisters and bubbles on the surface (figure 3a), with blisters increasing in size as a function of fluence. Each sample was then exposed to 100 pulses at either 40,33 or $5 \mathrm{MW} \mathrm{m}^{-2} \mathrm{~s}^{1 / 2}$ per pulse. After laser exposure no changes are observed for the case of $5 \mathrm{MW} \mathrm{m} \mathrm{m}^{-2} \mathrm{~s}^{1 / 2}$ pulses, while strong surface roughening is observed for all $33 \mathrm{MW} \mathrm{m} \mathrm{m}^{-2} \mathrm{~s}^{1 / 2}$ samples (a typical example is shown in figure $3 \mathrm{c}$ ). For the case of $40 \mathrm{MW} \mathrm{m} \mathrm{m}^{-2}$ pulses all samples show some reflow and recrystallisation as well as roughening with again no variation as a function of fluence. Comparison with a virgin target subjected to 100 pulses at $33 \mathrm{MW} \mathrm{m}^{-2} \mathrm{~s}^{1 / 2}$ shows almost no surface modification, unlike the pre-exposed targets (figure 3b). This therefore indicates a clear enhancement of damage caused by the prior plasma exposure. However, no clear difference in surface morphology was observed between samples exposed to different fluences. Previous literature [17] suggests that the roughening threshold is expected to reduce as mass loss and deuterium implantation increases with a $\Phi^{1 / 3}$ dependence. A reduction in near surface heat capacity or thermal conductivity due to the high implanted deuterium content in combination with rapid heating 
and bursting of subsurface deuterium-filled blisters was proposed to explain this observation [17]. In these experiments QMS measurements indicate that $30 \%$ of the surface deuterium inventory is released after the first pulse at $20 \mathrm{MW} \mathrm{m}^{-2} \mathrm{~s}^{1 / 2}$, rising to $70 \%$ at $40 \mathrm{MW} \mathrm{m} \mathrm{s}^{-2}$, while further releases never reach values higher than $10 \%$ of the initial pulse. By applying either one or five pulses instead of 100 to other samples it was found that the surface roughening increases gradually, indicating that the lack of fluence dependence could therefore be due to the immediate reduction of the surface deuterium after a single pulse. SEM analysis also showed no burst blisters in this case, as would be expected from a comparison with the experiments of Umstadter et. al. [17]. This would therefore indicate that the continual surface loading of the samples in simultaneous steady state and transient experiments could lead to the enhancement of damage.

A further sample was exposed to a constant fluence of $7.3 \times 10^{25} \mathrm{D}^{+} \mathrm{m}^{-2}$ and then subject to 100 pulses with a heat flux parameter between 20 and $65 \mathrm{MW} \mathrm{m}^{-2} \mathrm{~s}^{1 / 2}$ at differing places on its surface (see figure 4). As expected surface modification increases with applied laser power. Only surface roughening occurs at heat flux parameters of $30 \mathrm{MW} \mathrm{m} \mathrm{s}^{-2}$, recrystallisation and reflow occur at $40 \mathrm{MW} \mathrm{m}^{-2} \mathrm{~s}^{1 / 2}$, while cracking (and melting) only occurs for laser powers producing $60 \mathrm{MW} \mathrm{m}^{-2} \mathrm{~s}^{1 / 2}$ pulses, above the observed and expected melting threshold of $50 \mathrm{MW} \mathrm{m} \mathrm{m}^{-2}$. All exposures are summarised in figure 4. Similar electron beam experiments at JUDITH 1 observed cracking after 100 pulses at $30 \mathrm{MW} \mathrm{m}^{-2} \mathrm{~s}^{1 / 2}$ [18] which suggests that the lower energy threshold for cracking could be specific to particle loading or specific to electron guns, while the grade of tungsten could also play a role. The cracking threshold observed here also far exceeds that observed in section 3 which again indicates a possibility for combined effects to be important.

In order to further test the effect of synergistic enhancement of damage a target was exposed to simultaneous steady state plasma and pulsed laser irradiation in Magnum-PSI. The 
plasma conditions were similar to those of the pre-exposed targets above, although for a longer exposure time (115 s compared to $40 \mathrm{~s}$ ), while the laser produced a heat flux parameter of $20 \mathrm{MW} \mathrm{m}^{-2} \mathrm{~s}^{1 / 2}$ for each of 1150 pulses at a $10 \mathrm{~Hz}$ repetition rate. The results (figure $3 \mathrm{~d}$ ) show strong cracking of the surface. This suggests there is possibly a much lower threshold for cracking ( $\leq 20 \mathrm{MJ} \mathrm{m}^{-2} \mathrm{~s}^{1 / 2}$ here compared to $\sim 60 \mathrm{MJ} \mathrm{m}^{-2} \mathrm{~s}^{1 / 2}$ for the consecutive exposure case), further outlining the possible strong role of mobile hydrogen during the plasma exposure. However, direct comparison is not possible due to the differences in methodology, and the effect of pulse number on the surface damage in this case has not yet been quantified, as the increased damage could also be due to the higher number of pulses.

\section{Conclusions}

A combined steady state and pulsed plasma device has been developed at Pilot-PSI which can produce both a steady state flux and a transient heat and particle energy deposition of up to $1 \mathrm{MJ} \mathrm{m}^{-2}$. This system was used to expose a series of targets to increasing pulse power densities and differing pulse numbers. Surface roughening was observed after 10 pulses at $0.07 \mathrm{MJ} \mathrm{m}^{-2}$. This indicates a combined effect between steady and pulsed plasmas could enhance the rate of surface modification, possibly due to the increased levels of deuterium loading of the surface.

Pulsed laser irradiation of pre-exposed targets was made in order to develop an understanding of the effects of transient heating alone without simultaneous particle bombardment. Surface roughening was observed but only at higher heat flux parameters than for the case of section 2. The observed damage shows no dependence on pre-irradiation fluence which by comparison with [17] suggests that dynamic loading of hydrogen onto the surface may play an important role in damage to the surface. Furthermore the cracking 
threshold for the laser irradiated pulses following pre-exposure to plasma is higher than that for either of the two cases of exposure to simultaneous transient and steady state plasmas (section 3) and exposure to simultaneous transient laser and steady state plasmas (section 4), again suggesting a possible synergistic enhancement of damage, although differing pulse numbers could also be a significant factor.

A direct comparison between the two methodologies is not currently possible, therefore a high repetition rate pulsed plasma system, the Magnum ELM Simulation System, based on a capacitor bank design similar to the Pilot-PSI source, should be completed during summer 2012 [14]. This will allow exposure to identical steady state plasma conditions and either laser or pulsed plasma transients to be applied in a manner which can further disambiguate the effect of particle bombardment of the surface from heating alone. 


\section{Bibliography}

[1] R. Pitts, A. Kukushkin, A. Loarte, A. Martin, M. Merola, C. Kessel, V. Komarov and M. Shimada, Phys. Scr. , vol. T138, p. 014001, 2009.

[2] A. Loarte et. al., Phys. Scr., vol. T128, p. 222, 2007.

[3] K. Tokunaga, M. Baldwin, R. Doerner, N. Noda, Y. Kubota, N. Yoshida, T. Sogabe, T. Kato and B. Schedler, J. Nucl. Mater., Vols. 337-339, p. 887, 2005.

[4] S. Kajita, W. Sakaguchi, N. Ohno, N. Yoshida and T. Saeki, Nucl. Fusion, vol. 49, p. 095005, 2009.

[5] G. De Temmerman, K. Bystrov, R. Doerner, L. Marot, G. Wright, K. Woller, D. Whyte and J. Zielinski, these proceedings.

[6] J. Hanna, R. Doerner, R. Hernandez, R. Seraydarian and R. Pugno, Rev. Sci. Instrum., vol. 77, p. 123503, 2006.

[7] S. Djurović, Z. Mijatović, R. Kobilarov and I. Savić, Plasma Sources Sci. Technol., vol. 21, p. $025007,2012$.

[8] W. Vijvers, A high flux cascaded arc hydrogen plasma source (PhD thesis, Technische Universiteit Eindhoven), 2011.

[9] J. Westerhout et. al., Phys. Scr., vol. T128, pp. 18-22, 2007.

[10] J. Zielinski, R. Al, H. van der Meiden, W. Melissen, J. Rapp and G. De Temmerman, J. Nucl. Mater., vol. 415, pp. S70-S73, 2011.

[11] G. De Temmerman, J. Zielinski, H. van der Meiden, W. Melissen and J. Rapp, Appl. Phys. Lett., vol. 97 , p. 3, 2010.

[12] A. Loarte et. al., Nuclear Fusion, vol. 47, pp. S203-263, 2007.

[13] G. De Temmerman, J. Zielinski, S. van Diepen, L. Marot and M. Price, Nucl. Fusion, vol. 51, p. $073008,2011$.

[14] J. Rapp et. al., Fus. Eng. Des., vol. 85, pp. 1455-1459, 2010.

[15] S. Kajita, D. Nishijima, N. Ohno and S. Takamura, J. Appl. Phys., vol. 100, no. 10, p. 103304, 2006.

[16] S. Kajita, N. Ohno, S. Takamura, W. Sakaguchi and D. Nishishima, Appl. Phys. Lett., vol. 91, no. 26, p. 261501, 2007.

[17] K. Umstadter, R. Doerner and G. Tyan, Nucl. Fusion, vol. 51, no. 5, p. 053014, 2011. 
[18] M. Wirtz, J. Linke, G. Pintsuk, J. Rapp and G. Wright, J. Nucl. Mater., vol. 420, no. 11, pp. 218221, 2012. 
Figure 1: Evolution of the peak electron temperature (squares) and peak electron density (circles) measured $4 \mathrm{~cm}$ downstream from the plasma source as a function of peak input current.

Figure 2: SEM pictures of tungsten samples exposed to steady state plasma (a) or combined steady state and 10 pulsed plasmas of increasing energy densities from $0.07 \mathrm{MJ} \mathrm{m}^{-2}$, (b) 0.15 $\mathrm{MJ} \mathrm{m}^{-2}(\mathrm{c})$ and $0.5 \mathrm{MJ} \mathrm{m}^{-2}(d)$.

Figure 3: SEM pictures of tungsten samples exposed to either (a) only 40s steady state plasma (fluence $\left.2.5 \times 10^{26} \mathrm{~m}^{-2}\right)$, (b) only transient laser pulses (10033 $\mathrm{MW} \mathrm{m}^{-2} \mathrm{~s}^{1 / 2}$ pulses) (c) consecutive exposures of $(a)$ and $(b)$ or $(d)$ simultaneous exposures of $115 \mathrm{~s}$ steady state plasma and $115020 \mathrm{MJ} \mathrm{m}^{-2} \mathrm{~s}^{1 / 2}$ pulses.

Figure 4: Summary of laser irradiation experiments performed giving damage to surface as a function of heat flux parameter to the surface and pre-exposure fluence to the target. All targets subjected to 100 pulses. 


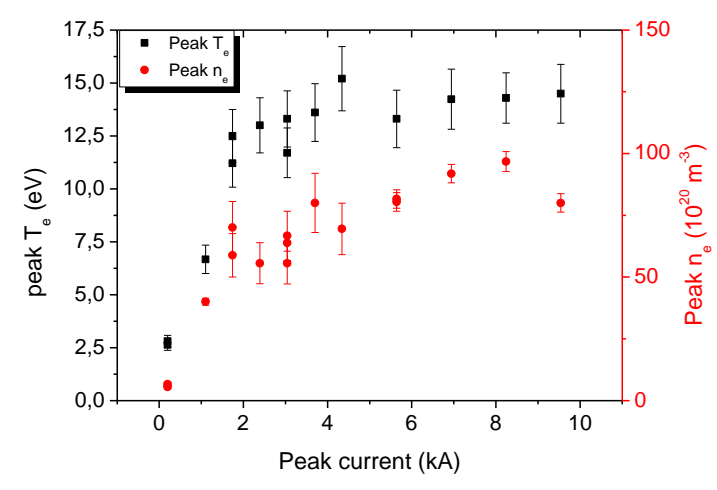

Figure 1 


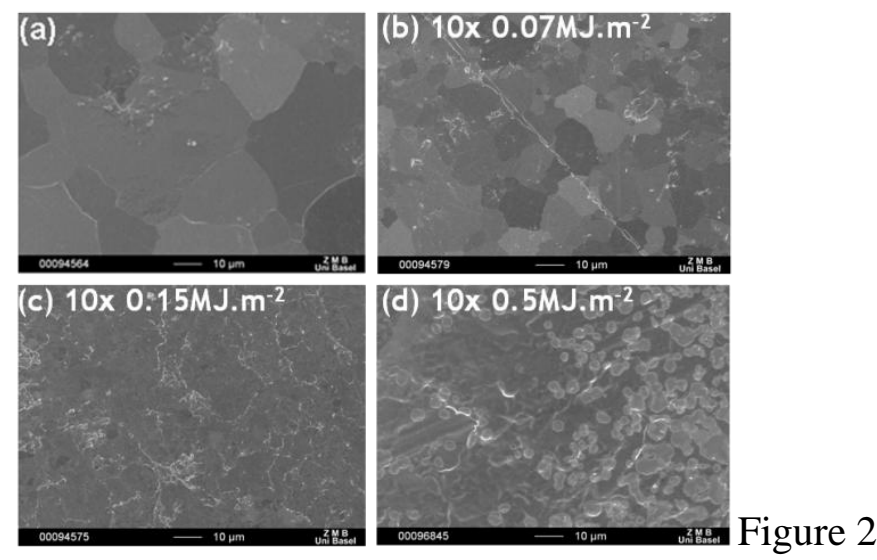




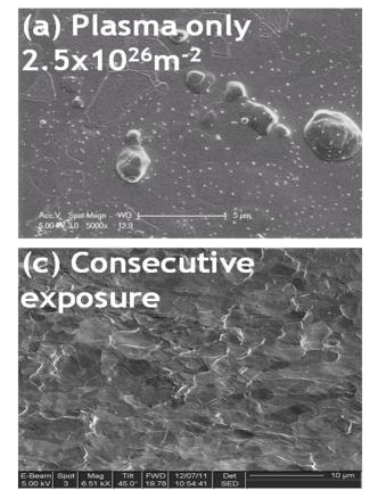

(b) Laser only

(33 $\left.\mathrm{MW} \mathrm{m}^{-2} \mathrm{~s}^{1 / 2}\right)$

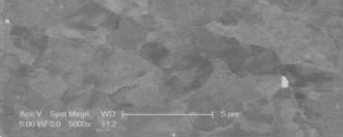

(c) Simutanesus

exposure

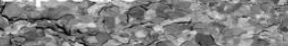

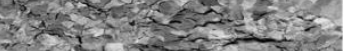

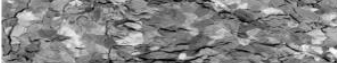

Figure 3 


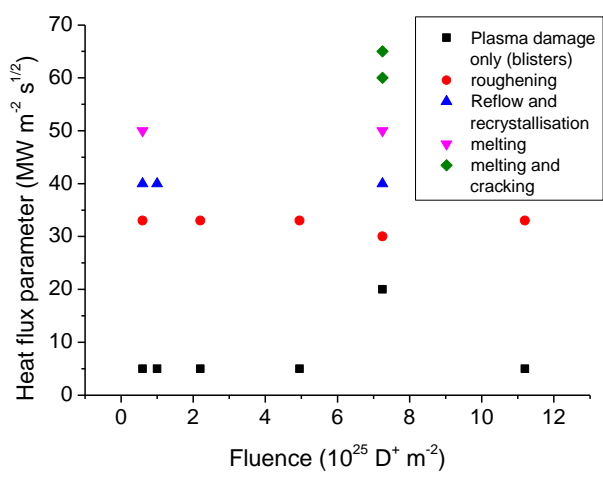

Figure 4 